\title{
Management Infertility by Hormones Replacement Therapy in Women with Polycystic Ovarian Syndrome
}

\author{
Sardar Qadir Omer, Tariq Waece Sadiq* \\ Department of Pharmacy, Erbil Technical Institute, Erbil Polytechnic University, Erbil, Kurdistan Region, Iraq
}

\author{
*Corresponding author: \\ Tariq Waece Sadeq, \\ Department of Pharmacy, \\ Erbil Technical Institute, Erbil \\ Polytechnic University, Erbil, \\ Iraq. E-mail: tariq.sadiq@ \\ epu.edu.iq
}

Received: 12 March 2019

Accepted: 23 October 2019

Published: 30 June 2020

\section{DOI}

10.25156/pti.v10n1y2020.pp170-174

\section{A B S T R A C T}

Polycystic ovary syndrome (PCOS) is an endocrine-metabolic disorder characterized by multiple hormonal imbalances; the clinical presentation dominated by manifestations of hyperandrogenism, which generates short- and long-term consequences on female health sterility, infertility is one of the most alarming associated morbidities. Forty women (28.9 \pm 0.8 years old) with polycystic ovarian syndrome had infertility and there were enrolled in these clinical trials and randomly allocated into two groups. Group one and Group two were given treated infertility drugs (Clomid $5 \mathrm{mg}$ for 5 days) and recombinant follicle-stimulating hormone ( $\mathrm{rFSH}) 75 \mathrm{IU}$ for long and short duration to get pregnant. Serum testosterone, progesterone, follicle-stimulating hormone (FSH), and luteinizing hormone (LH) of both groups were measured when women diagnosed as PCOS. The results showed that women which treated by low-dose recombinant FSH 75 IU for 3 days consequently (Group one) have significant decreased LH, while progesterone and FSH significantly increased after treated by hormone therapy $P \geq 0.05$ while after treatment with recombinant FSH IU for 5 days (Group two), the result showed non-significant effect as compared with the first group. Concluded: Increase pregnant rate and decrease over stimulation syndrome among infertile women associated with polycystic overian syndrome, when treated with applicated low dose and short term by rFSH hormone

Keywords: Infertility; Polycystic ovary syndrome; Follicle-stimulating hormone; Luteinizing hormone, Progesterone

\section{INTRODUCTION}

Polycystic ovary syndrome (PCOS) is characterized to be a metabolic syndrome which may include hyperinsulinemia, hyperlipidemia, diabetes mellitus, and possible cardiac disease, as well as high androgen levels, anovulation, infertility, endometrial cancer, and obesity (Norman, 2002; Lobo and Carmina, 2000). The estrogens stimulate luteinizing hormone $(\mathrm{LH})$ and inhibit follicle-stimulating hormone (FSH) secretion, contributing to granulosa cell and theca cell (TC) hyperplasia in obese women with PCOS; this would increase androgen synthesis and reinforcing this cycle of hyperestrogenemia-hyperandrogenemia in obese women with this pathology which is serving as substrates for extraovarian aromatization (Pasquali and Casimirri, 1993). Feedback disturbances in the hypothalamus-hypophysis-ovary axis are another typical feature of PCOS (Blank et al., 2006). That they suppress endogenous FSH levels, thereby preventing maturation of the small "healthy" follicles (Chavez-Ross et al., 1997). Ovary produces inappropriate levels of estradiol (and progesterone) for their size (Willis et al., 1998). Higher levels of gonadotropin-releasing hormone $(\mathrm{GnRH})$ induce greater androgen synthesis in ovarian TC due to an increased frequency and amplitude of GnRH and LH pulsatile secretion (Blank et al., 2009).

In PCOS, the ovaries may not release follicle regularly or they may not release health follicle. High levels of antiMullerian hormone are appeared to play an important role in long-term disruption of ovarian physiology in women with PCOS (Karkanaki et al., 2011). Elevated levels AMA concentration associated with infertility (Pierre et al., 2013). Infertility is currently affects the women aged 20-44 years, as it one of the most alerting associated morbidity (Mascarenhas et al., 2012). Failure to become pregnant without using contraception after 1 year of regular intercourse in women $<35$ years and after 6 months in women $>$ years is called infertility (Practice Committee of the American Society for Reproductive Medicine, 2008).

Progesterone is essential for the implantation and maintenance of early human pregnancy. The follicular phase of the menstrual cycle is estrogen dominated, while the luteal phase of the menstrual cycle is progesterone dominated (Cameron et al., 1996). Progesterone is produced after ovulation by the corpus luteum (sack that the egg comes from) and dominates the second half 
of the cycle (luteal phase). In many species, a decrease in circulating progesterone at the time of fertilization is sufficient to delay implantation so the progesterone has a major role in the endometrium in preparation for implantation of a fertilized ovum (Rothchild, 1983). In women with PCOS, ovarian follicles arrest in a state of pre-ovulation; this state of arrest is caused primarily by an overabundance of androgens, which impedes follicle growth and ovulation. Current opinion suggests that insulin resistance (IR) is the culprit because it is observed in many women with PCOS (Dunaif et al., 1989; Johnson, 2014). Causes an abnormal response in the ovary that results in an increase in the amount of circulating androgens is IR that leads to hyperandrogenism (Fritz et al., 2011).

Female infertility can result from age, physical problems, hormone abnormality, and lifestyle or environmental factors. Most cases of infertility in women result from problems with producing eggs. In premature ovarian failure, the ovaries stop functioning before natural menopause. Therefore, considering the severe consequences, PCOS exerts on the health and lifestyle of the affected women, it is most importance to unravel the intricate pathophysiologic cross-talk among PCOS, IR, and obesity. Aside from disturbances in insulin physiology, obesity implies thorough alterations in steroid hormone metabolism, essentially summarized as increased concentrations of nearly all of these messengers. GnRH agonist $(\mathrm{GnRHa})$ is effective even severe IR in women with PCOS who are unresponsive to oral contraceptive; the GnRHa suppresses pituitary hormones, decreases androgen and estradiol secretion, and improves severe forms of hirsutism (Cristello et al., 2005).

\section{Aim of Study}

The aim of the study is to decrease percentage of infertility in women with polycystic ovarian syndrome and study the effects of various duration of dose hormone recombinant FSH ( $\mathrm{rFSH}$ ) on ovary and women hormone.

\section{MATERIALS AND METHODS}

This study was carried out from 2016 to 2017 and this was included 40 women with an ovulatory infertility due to PCOS were taken randomly which was diagnosed by gynecologist in the Erbil Maternal Hospital, the mean $( \pm$ SE) age of the patients was $28.9 \pm 0.8$ years, and their mean duration of infertility was $2.2 \pm 0.2$ years. All patients had both ovaries and had to ovulate with clomiphene citrate at doses of $100 \mathrm{mg} /$ day after regulation menstruation cycle with oral contraceptive Progyluton. Forty women healthy were taken in this study as a control.

\section{Protocol Treatment}

Women infertile with PCOS were divided in two groups, Group one was treated with low-dose (Gonal F) FSH subcutaneously injection 75 IU for 3 days while Group two treated with high-dose $\mathrm{rFSH}$ subcutaneously injection $75 \mathrm{IU}$ for 5 days. Sonography was done for two groups to determined size follicles in ovaries at on human chorionic gonadotropin (HCG) day and check serum level hormone FSH, LH, progesterone, and testosterone. These tests were done by Minivid apparatus and used Minivid kits.

\section{Blood Sample}

Blood samples were drawn from each patients with PCOS, and the serum was utilized for determination level hormones, follicle stimulation hormone, LH, progesterone, and testosterone. These investigations repeated after treated with hormone replacement on HCG.

Patients with irregular menstruation by lack hormone progesterone level are participating in this study and any other defects infertility women with PCOS which are excluded.

\section{Statistical Analysis}

Analysis of variance (ANOVA) was used to find the differences between groups. The Duncan test was applied to determined factor effect. $P$ value was considered significant, when it is less than 0.05 .

\section{RESULTS}

The result of our study was revealed that the serum levels (FSH, LH, and progesterone) of women with PCOS in Group 1 (after treatment with low dose $75 \mathrm{IU}$ of $\mathrm{rFSH}$ ) were significantly changed on day HCG while testosterone level was decrease but no significant change at $P>0.05$ when compared with control group, as shown in Table 1.

After treatment, 20 infertile women with PCOS (Group 2) with long-term therapy (75 IU rFSH), the serum levels (FSH and progesterone) were significantly increase but the serum level LH was significant decrease while testosterone was no significant change at $P>0.05$ when compared with before treated, as shown in Table 2.

As shown in Table 3, the women with PCOS treated short term by $75 \mathrm{IU}$ that pregnancy rate was high and ovary hyperstimulation syndrome was less occurs in Group 1 as compared with Group 2 women with PCOS with longterm therapy.

\section{DISCUSSION}

Polycystic ovary disease (PCOS) is a hormonal imbalance in women that are thought to be one of the leading causes 
of female infertility (Hack et al., 1972). PCOS causes of an ovulatory infertility approximately $75 \%$ of cases (Goldenberg and Glueck, 2008). The result of this study was showed management women infertility with polycystic ovarian syndrome which is characterized hormone disturbance progesterone, testosterone, FSH, and LH compared with control. This oligomenorrhea was treated by oral contraceptive which is contained progesterone and estrogen. Menstrual irregular management using progesterone replacement is beginning to treat infertility, it is similar to studies along with women PCOS complaints of menstrual irregularity, the often chronic an ovulation that is associated with PCOS can increase patients infertility and inhibited endometrial proliferation. Using ether cyclic progestin or a low-dose contraceptive was containing estrogen and progestin (Gorry et al., 2006). The primary recommended treatment option for PCOS-related menstrual disorders and improved menstrual regularity with low-dose contraceptive (Badawy and Elnashar, 2011). PCOS affects approximately $5-10 \%$ of women of

\begin{tabular}{|c|c|c|c|}
\hline \multirow{3}{*}{$\begin{array}{l}\text { Level serum } \\
\text { hormone }\end{array}$} & Control & Group1(PCOS) & Group1 (PCOS) \\
\hline & \multirow[t]{2}{*}{$n=20$} & Before treatment & On day (HCG) \\
\hline & & $n=20$ & $n=20$ \\
\hline $\mathrm{FSH}$ & $5.17 \pm 0.55$ & $3.90 \pm 0.52^{*}$ & $5.54 \pm .58$ \\
\hline LH & $5.66 \pm 0.90$ & $14 \pm 2.41^{*}$ & $6.42 \pm .98$ \\
\hline Progesterone & $4.83 \pm 0.59$ & $0.44 \pm 0.18^{*}$ & $4.64 \pm .56$ \\
\hline Testosterone & $0.29 \pm 0.13$ & $0.85 \pm .13$ & $0.67 \pm 0.14$ \\
\hline Ratio LH:FSH & 1:01 & 3:01 & 1:01 \\
\hline \multicolumn{4}{|c|}{$\begin{array}{l}\text { Data represented by mean } \pm \mathrm{SD} . \mathrm{N}=\text { number of subjects, significant } \\
\text { difference }{ }^{*} \text {, significant difference at level } P<0.05\end{array}$} \\
\hline \multicolumn{4}{|c|}{$\begin{array}{l}\text { Table 2: Measurement serum level hormone (FSH, LH, } \\
\text { progesterone, and free testosterone) in infertile women with } \\
\text { PCOS before and after r FSH hormone therapy for } 5 \text { days }\end{array}$} \\
\hline \multirow{3}{*}{$\begin{array}{l}\text { Level serum } \\
\text { hormone }\end{array}$} & Control & Group 2 (PCOS) & Group 2 (PCOS) \\
\hline & $n=20$ & Before treatment & On day (HCG) \\
\hline & & $n=20$ & $n=20$ \\
\hline $\mathrm{FSH}$ & $5.17 \pm 0.55$ & $3.89 \pm 0.50^{*}$ & $6.64 \pm 1.82$ \\
\hline LH & $5.66 \pm 0.90$ & $13.48 \pm 1.05^{*}$ & $6.31 \pm 0.84$ \\
\hline Progesterone & $4.83 \pm 0.59$ & $0.49 \pm 0.21^{*}$ & $4.67 \pm 0.68$ \\
\hline Free testosterone & $0.29 \pm 0.13$ & $0.88 \pm 0.17$ & $0.72 \pm 0.16$ \\
\hline Ratio LH:FSH & 1:01 & 3:01 & 1:01 \\
\hline
\end{tabular}

data represented by mean $\pm \mathrm{sd}, n=$ number of subjects, significant difference ${ }^{*}$, significant difference at level $p<0.05$ reproductive age and is one of the most common causes of anovulatory infertility (ACOG Committee on Practice Bulletins - Gynecology ACOG Practice Bulletin No 108. 2009). Menstrual disruption typically manifests in PCOS, ranging from oligomenorrhea to amenorrhea.

Both groups (1 and 2) were associated with low FSH (3.90 $\pm 0.52),(3.89 \pm 0.50)$ and high level LH (14 \pm 2.41$),(13.48$ $\pm 1.05)$, as shown in Tables 1 and 2, hormonal abnormality in women with PCOS leads to an ovulation and infertility. This is right with these studies elevated LH concentrations frequently are encountered in patients with PCOS and different studies have linked excessive LH secretion with detrimental effects on reproductive function, such as irregular menstrual cycles, an ovulation, infertility, and miscarriage (Hopkinson et al., 1998).

In our study women infertile with PCOS associated with hyperandrogenism, which lead imbalance serum hormones that elevated LH and decrease growth follicles in ovaries which lead to infertility. This result was coherent that Gonadotropin especially elevated in luteinizing hormone (Brothers., et al 2010). Polycystic ovary syndrome accounts for more than $75 \%$ of cases of anovulatory infertility. The mechanism of anovulation is uncertain but there is evidence that arrested antral follicle development is associated with the abnormal endocrine profile, in particular the interaction of insulin and LH on granulosa cell differentiation.(Gorry., et al 2006).

In Tables 1 and 2, women with polycystic ovarian syndrome were associated with increase serum level testosterone or androgen hormone this is right with these studies more than $80 \%$ of women presenting with symptoms of androgen excess have PCOS (Qiao and Feng, 2010). Hirsutism is a common clinical presentation of hyperandrogenism occurring in up to $70 \%$ of women with PCOS (Filicori, 1999).

Results revealed non-significant statistical differences $(P>0.005)$ at high-dose and low-dose recombinant FSH subcutaneous injection on pregnancy rate in PCOS patients. However, management anovulatory in Group one women with PCOS by low-dose rFSH was better than high-dose rFSH in Group two, but in Group one,

Table 3: Comparison effects of long-term therapy and short-term therapy recombinant rFSH hormone therapy in women infertility with polycystic ovarian syndrome

\begin{tabular}{lcccc} 
& Group 1 & Group 2 & Percentage & Percentage \\
\cline { 3 - 5 } & & & Group 1 & Group 2 \\
Ovarian hyperstimulation syndrome (OHSS) & 1 & 4 & 70 & 20 \\
Hirsutism (facial) & 14 & 3 & $30 \%$ & 75 \\
Pregnancy rate & 6 & $>20 \mathrm{~mm}$ & & 15 \\
Size of follicle on day HCG & $<14 \mathrm{~mm}$ & & \\
\hline
\end{tabular}


ovarian hyperstimulation syndrome (OHSS) less occurs and pregnancy rate was high. This was confirmed with studies the goal of FSH administration for ovulation induction which is the development of a single follicle resulting in a singleton live birth. Risks associated with ovulation induction include OHSS and pregnancy with multiples. Because women with PCOS are very sensitive to the effects of FSH, a low-dose step-up protocol is recommended (Taymar, 1996; Thessaloniki, 2008). For women with PCOS, treatment a low-dose regimen of gonadotropins $\mathrm{rFSH}$ has almost completely eliminated the complication of OHSS and maintaining an acceptable pregnancy rate (Christin-Maitre and Hugues, 2003).

\section{CONCLUSION}

According to this study, we conclude that decreased infertility women with polycystic ovarian syndrome after treated with low-dose recombinant FSH therapy associated increase pregnancy rate and less ovary hyperstimulation syndrome OHSS than high-dose recombinant FSH therapy.

\section{RECOMMENDATIONS}

1. Management infertility in women with polycystic ovarian syndrome by other hormone drugs

2. Comparative study with women polycystic ovarian syndrome treated by metformin and with weight by exercise

3. Comparative study between infertility women with polycystic ovarian syndrome treated by hormone with ovary drill by laparoscopy.

\section{REFERENCES}

Badawy, A. and A. Elnashar. 2011. Treatment options for polycystic ovary syndrome. Int. J. Womens Health. 3: 25.

Blank, S. K., C. R. McCartney and J. C. Marshall. 2006. The origins and sequelae of abnormal neuroendocrine function in polycystic ovary syndrome. Hum. Reprod. Update. 12(4): 351-361.

Blank, S. K., C. R. McCartney, S. Chhabra, K. D. Helm, C. A. Eagleson, R. J. Chang and J. C. Marshall. 2009. Modulation of gonadotropinreleasing hormone pulse generator sensitivity to progesterone inhibition in hyperandrogenic adolescent girls-implications for regulation of pubertal maturation. J. Clin. Endocrinol. Metab. 94(7): 2360-2366.

Brothers, K. J., S. Wu, S. A. DiVall, M. R. Messmer, C. R. Kahn, R. S. Miller, S. Radovick, F. E. Wondisford and A. Wolfe. 2010. Rescue of obesity-induced infertility in female mice due to a pituitary-specific knockout of the insulin receptor. Cell Metab. 12(3): 295-305.

Cameron, I. T., G. Irvine and J. E. Norman. 1996. Menstruation. In: Hiller, S. G., H. C. Kitchener and J. P. Neilson, editors. Scientific Essentials of REPRODUCTIVE MEDICINE. W.B. Saunders, London.
Chavez-Ross, A., S. Franks, H. D. Mason, K. Hardy and J. Stark. 1997. Modelling the control of ovulation and polycystic ovary syndrome. J. Math. Biol. 36(1): 95-118.

Christin-Maitre, S. and J. N. Hugues. 2003. A comparative randomized multicentric study comparing the step-up versus step-down protocol in polycystic ovary syndrome. Hum. Reprod. 18(8): 1626-1631.

Cristello, F., V. Cela, P. G. Artini and A. R. Genazzani. 2005. Therapeutic strategies for ovulation induction in infertile women with polycystic ovary syndrome. Gynecol. Endocrinol. 21(6): 340-352.

Dunaif, A., K. R. Segal, W. Futterweit and A. Dobrjansky. 1989. Profound peripheral insulin resistance, independent of obesity, in polycystic ovary syndrome. Diabetes. 38(9): 1165-1174.

Filicori, M. 1999. The role of luteinizing hormone in folliculogenesis and ovulation induction. Fertil. Steril. 71(3): 405-414.

Fritz, M. A., L. Speroff and M. J. del Sol Jaquotot. 2011. Endocrinología Ginecológica Clínica y Esterilidad. Wolters Kluwer Health, Lippincott Williams \& Wilkins, Philadelphia, PA.

Goldenberg, N. and C. Glueck. 2008. Medical therapy in women with polycystic ovarian syndrome before and during pregnancy and lactation. Minerva Ginecol. 60(1): 63-75.

Gorry, A., D. M. White and S. Franks. 2006. Infertility in polycystic ovary syndrome. Endocrine. 30(1): 27-33.

Hack, M., M. Brish, D. M. Serr, V. Insler, M. Salomy and B. Lunenfeld. 1972. Outcome of pregnancy after induced ovulation: Followup of pregnancies and children born after clomiphene therapy. JAMA. 220(10): 1329-1333.

Hopkinson, Z. E., N. Sattar, R. Fleming and I. A. Greer. 1998. Polycystic ovarian syndrome: The metabolic syndrome comes to gynaecology. BMJ. 317(7154): 329-332.

Johnson, N. P. 2014. Metformin use in women with polycystic ovary syndrome. Ann. Transl. Med. 2(6): 56.

Karkanaki, A., C. Vosnakis and D. Panidis. 2011. The clinical significance of anti-Müllerian hormone evaluation in gynecological endocrinology. Hormones. 10(2): 95-103.

Lobo, R. A. and E. Carmina. 2000. The importance of diagnosing the polycystic ovary syndrome. Ann. Intern. Med. 132(12): 989-993.

Mascarenhas, M. N., S. R. Flaxman, T. Boerma, S. Vanderpoel and G. A. Stevens. 2012. National, regional, and global trends in infertility prevalence since 1990: A systematic analysis of 277 health surveys. PLoS Med. 9(12): e1001356.

Morgante, G., R. Orvieto, A. Di Sabatino, M. C. Musacchio and V. De Leo. 2011. The role of inositol supplementation in patients with polycystic ovary syndrome, with insulin resistance, undergoing the low-dose gonadotropin ovulation induction regimen. Fertil. Steril. 95: 2642-2644.

Norman, R. J. 2002. Hyperandrogenaemia and the ovary. Mol. Cell. Endocrinol. 191(1): 113-119.

Pasquali, R. and F. Casimirri. 1993. The impact of obesity on hyperandrogenism and polycystic ovary syndrome in premenopausal women. Clin. Endocrinol. 39(1): 1-16.

Pierre, A., M. Peigné, M. Grynberg, N. Arouche, J. Taieb, L. Hesters, J. Gonzalès, J. Y. Picard, D. Dewailly, R. Fanchin and S. Catteau-Jonard. 2013. Loss of LH-induced down-regulation of anti-Müllerian hormone receptor expression may contribute to anovulation in women with polycystic ovary syndrome. Hum. Reprod. 28(3): 762-769.

Practice Committee of the American Society for Reproductive Medicine. 2012. Diagnostic evaluation of the infertile female: A committee opinion. Fertil. Steril. 98(2): 302-307. 
Qiao, J. and H. L. Feng. 2011. Extra-and intra-ovarian factors in polycystic ovary syndrome: Impact on oocyte maturation and embryo developmental competence. Hum. Reprod. Update. 17(1): 17-33.

Rothchild, I. 1983. Role of progesterone in initiating and maintaining pregnancy. In: Progesterone and Progestins. Raven Press, New York. p219-229.

Shoham, Z., H. S. Jacobs and V. Insler. 1993. Luteinizing hormone: Its role, mechanism of action, and detrimental effects when hypersecreted during the follicular phase. Fertil. Steril. 59(6): 1153-1161.
Taymar, M. I. 1996. Regulation of follicle growth: some clinical implications in reproductive endocrinology. Fertil. Steril. 65: 235247.

Thessaloniki, T. 2008. PCOS consensus workshop group consensus on infertility treatment related to polycystic ovary syndrome. Fertil. Steril. 89: 505-522.

Willis, D. S., H. Watson, H. D. Mason, R. Galea, M. Brincat and S. Franks. 1998. Premature response to luteinizing hormone of granulosa cells from anovulatory women with polycystic ovary syndrome: Relevance to mechanism of anovulation. J. Clin. Endocrinol. Metab. 83(11): 3984-3991. 